\title{
Testing foundations of modern cosmology with SKA all-sky surveys
}

\author{
Dominik J. Schwarz ${ }^{*}{ }^{1}$ David Bacon, ${ }^{2}$ Song Chen, ${ }^{1}$ Chris Clarkson, ${ }^{3}$ Dragan \\ Huterer, ${ }^{4}$ Martin Kunz, ${ }^{5,6}$ Roy Maartens, ${ }^{7,2}$ Alvise Raccanelli, ${ }^{8,9,10}$ Matthias Rubart, ${ }^{1}$ \\ Jean-Luc Starck ${ }^{11}$ \\ ${ }^{1}$ Fakultät für Physik, Universität Bielefeld, 33501 Bielefeld, Germany; ${ }^{2}$ Institute of Cosmology \\ \& Gravitation, University of Portsmouth, Portsmouth POI 3FX, United Kingdom; ${ }^{3}$ Centre for \\ Astrophysics, Cosmology \& Gravitation and Department of Mathematics \& Applied \\ Mathematics, University of Cape Town, Cape Town 7701, South Africa; ${ }^{4}$ Department of \\ Physics, University of Michigan, Ann Arbor, MI 48109-1040, USA; ${ }^{5}$ Université de Genève, \\ Département de Physique Théorique and CAP, CH-1211 Genève 4, Switzerland; ${ }^{6}$ African \\ Institute for Mathematical Sciences, Cape Town 7945, South Africa; ${ }^{7}$ Physics Department, \\ University of the Western Cape, Cape Town 7535, South Africa; ${ }^{8}$ Jet Propulsion Laboratory, \\ California Institute of Technology, Pasadena CA 91109, USA $;{ }^{9}$ California Institute of \\ Technology, Pasadena CA 91125, USA; ${ }^{10}$ Department of Physics \& Astronomy, Johns Hopkins \\ University, 3400 N. Charles St., Baltimore, MD 21218, USA ${ }^{11}$ Laboratoire AIM, UMR \\ CEA-CNRS-Paris, Irfu, SAp, CEA Saclay, F-91191 Gif-sur-Yvette CEDEX, France \\ E-mail: dschwarzephysik.uni-bielefeld.de
}

\begin{abstract}
Continuum and HI surveys with the Square Kilometre Array (SKA) will allow us to probe some of the most fundamental assumptions of modern cosmology, including the Cosmological Principle. SKA all-sky surveys will map an enormous slice of space-time and reveal cosmology at superhorizon scales and redshifts of order unity. We illustrate the potential of these surveys and discuss the prospects to measure the cosmic radio dipole at high fidelity. We outline several potentially transformational tests of cosmology to be carried out by means of SKA all-sky surveys.
\end{abstract}

Advancing Astrophysics with the Square Kilometre Array

June 8-13, 2014

Giardini Naxos, Italy

\footnotetext{
* Speaker.
} 


\section{Introduction}

The Square Kilometre Array (SKA) will allow us to test fundamental assumptions of modern cosmology at redshifts of order unity and at an accuracy level matching and complementing the high fidelity observations of the cosmic microwave background (CMB).

The Cosmological Principle states that the Universe is spatially isotropic and homogeneous. This holds on sufficiently large scales and needs to be interpreted in a statistical sense. Historically, it provided a very powerful motivation to single out the Friedmann-Lemaitre models despite a lack of knowledge regarding the initial conditions of the Universe. Cosmological inflation, proposed in the 1980s, allowed the Universe to start from a reasonably small patch of almost homogeneous and isotropic space. According to the idea of cosmological inflation, suddenly at least one small patch is inflated to contain today's observable Universe. In the course of inflation, any previously existing anisotropy or inhomogeneity is exponentially diluted. However, unavoidable quantum fluctuations are squeezed by the rapid expansion during inflation and become the seeds for large scale structure. The result is a statistically isotropic and homogeneous Universe (at least locally).

These ideas are confirmed by the observed high degree of isotropy of CMB radiation, which enables us to define a CMB frame by measuring a temperature monopole, $T_{0}=2.2755 \pm 0.0006 \mathrm{~K}$ and dipole, $T_{1}=3.355 \pm 0.008 \mathrm{mK}$ towards $(l, b)=\left(263.99^{\circ} \pm 0.14^{\circ}, 48.26^{\circ} \pm 0.03^{\circ}\right)$ (Hinshaw et al. 2009). The concept of a spatially homogeneous Universe allows us to speak about a cosmic time or an age of the Universe. By measuring the CMB temperature $T_{0}$ and the present expansion rate of the Universe $H_{0}$ we can anchor the thermal history of the Universe to its expansion history.

Radio surveys played an important role to establish that the Universe extends to redshifts beyond unity and that it is almost isotropic [see e.g. Ryle \& Clarke (1961)]. Today, observations of the CMB confirm the predictions of cosmological inflation impressively (Planck collaboration 2014a; 2014b). However, it is unknown for how long (or how many e-foldings) inflation took place. In order to explain the observed spatial flatness of the Universe, about 50 to 60 e-foldings could be enough, but in many models it took much longer, i.e. the domain in which the statistical cosmological principle applies is expected to be much larger than the observable Universe. The quest to determine the duration of inflation, as well as the related question of the topology of the Universe, can only be answered by observing the biggest scales.

Interestingly enough, the $\mathrm{CMB}$ exhibits unexpected features at the largest angular scales, among them a lack of angular correlation, alignments between the dipole, quadrupole and octupole, hemispherical asymmetry, a dipolar power modulation, and parity asymmetries (Planck collaboration 2014c; Copi et al. 2013a; 2013b). Understanding the statistical significance of these anomalies is a hot topic (Bennett et al. 2013; Starck et al. 2013; Rassat et al. 2014) since lack of statistical isotropy or Gaussianity could rule out the standard cosmological model. As the precision of these CMB measurements is limited by our understanding of the foregrounds and observational uncertainties are already much smaller than the cosmic variance at those scales, it is very hard to identify the cause of these anomalies without an independent probe at the same scales.

However, based on the observed CMB anisotropies and despite of these anomalies, deviations from statistical isotropy have to be small. The observational situation is less clear for the case of statistical homogeneity, as testing the assumption of isotropy is much simpler than testing homogeneity (Maartens 2011; Clarkson 2012). 
SKA will probe an enormous number of independent modes when studying the large-scale structure of the Universe and will measure superhorizon sized modes at redshifts of order unity (better than any existing or planned infrared, optical, or X-ray campaign). This will enable us to probe scales that have not been in causal contact since the first horizon crossing during inflation and that contain information that was frozen in during cosmological inflation. In contrast to the $\mathrm{CMB}$, the radio sky provides a probe of those largest scales at a redshift of order unity (2D for continuum surveys and 3D for HI surveys).

SKA would enable several tests of the fundamental cosmological principles. For example, the rest frames of the CMB and large scale structure (LSS) may not coincide due to novel superhorizon physics - for example, presence of isocurvature modes (Erickcek et al. 2008). SKA's width and depth will enable a measurement of the kinematic dipole with respect to the LSS reference frame via the relativistic aberration and Doppler shift ("bunching up" of SKA sources in the direction of the dipole). This, when combined with the CMB's own measurement of the kinematic dipole would, for the first time, enable the test of whether the two reference frames - that of the CMB and the LSS - are one and the same, as demanded by the Cosmological Principle.

Here we describe how to use all-sky $(3 \pi)$ SKA continuum surveys to test statistical isotropy and to measure the cosmic dipole and other low- $\ell$ multipole moments. These issues are tightly connected to tests of non-Gaussianity and the topology of the Universe, the former aspect is described in Camera et al. (2015). All-sky SKA HI threshold surveys will additionally allow us to test the homogeneity of the Universe at superhorizon scales - a test that has never before been performed. Statistical homogeneity and isotropy are assumed to hold true in other cosmology-related contributions to this book (Bull et al. 2015; Raccanelli et al. 2015). Tests of statistical isotropy and homogeneity will also allow (and force) us to dig deep into the systematics of SKA surveys and thus help to put all cosmological and non-cosmological results of SKA surveys on firm grounds.

The conceptually simplest probe of cosmology is differential number counts (de Zotti et al. 2010). If no redshift information is available, one can count the number of (extragalactic) radio sources per solid angle and flux density. Besides flux calibration issues, the cosmological information contained in differential number counts is limited by the diversity of radio sources and their luminosity and density evolution. Radio sources fall into two principal classes, active galactic nuclei (AGN) and star forming galaxies (SFG). The exquisite angular resolution of SKA surveys will allow us to resolve most of the AGNs and thus to obtain an extra handle based on morphology. Another possibility to overcome the restrictions from evolution is to study the directional fluctuations of differential number counts (Raccanelli et al. 2012; Chen \& Schwarz 2014), as we do not expect that the properties of radio sources would single out preferred directions in the Universe.

All SKA forecasts presented in this work assume the baseline design and imaging capabilities as presented in Dewdney et al. (2013); Braun (2014).

\section{Cosmic radio dipole (Early Science, SKA1 \& SKA2)}

The CMB dipole is generally assumed to be due to our peculiar motion and thus defines a cosmic reference frame. However, the observation of the dipole in the microwave sky alone does not allow us to tell the difference between a motion-induced CMB dipole and dipole contributions from other physical phenomena [e.g. the model in Erickcek et al. (2008)]. 


\begin{tabular}{llll}
$\begin{array}{l}\text { SKA 3 } \pi \text { surveys } \\
\text { (centr. frequency, ang. res.) }\end{array}$ & Early Science & SKA1 & SKA2 \\
\hline LOW (151 MHz, 10") & $1.0 \times 10^{8}(20 \mu \mathrm{Jy})$ & $2.4 \times 10^{8}(10 \mu \mathrm{Jy})$ & $2.2 \times 10^{9}(1 \mu \mathrm{Jy})$ \\
MID/SUR B1 (610 MHz, 1") & $7.8 \times 10^{7}(10 \mu \mathrm{Jy})$ & $1.9 \times 10^{8}(5 \mu \mathrm{Jy})$ & $1.8 \times 10^{9}(0.5 \mu \mathrm{Jy})$ \\
MID/SUR B2 (1.4 GHz, 0.5”) & $3.8 \times 10^{7}(10 \mu \mathrm{Jy})$ & $9.7 \times 10^{7}(5 \mu \mathrm{Jy})$ & $1.2 \times 10^{9}(0.5 \mu \mathrm{Jy})$
\end{tabular}

Table 1: Expected total number of radio sources $(10 \sigma)$ in various frequency bands and survey instruments, assuming the SKA baseline design and the cosmology and differential number counts as simulated in Wilman et al. (2008). In order to match observations at $1.4 \mathrm{GHz}$, the number of SFG has been multiplied by a factor of 2.5 compared to the simulations for all frequency bands. The numbers in brackets denote the assumed rms noise levels.

Due to the effects of aberration and Doppler shift, the kinetic dipole must also be present in radio observations (Ellis \& Baldwin 1984). Besides the kinetic dipole, we also expect contributions from the large-scale structure and from Poisson noise. Such a radio dipole has been looked for in radio source catalogues, such as NVSS (Blake \& Wall 2002; Singal 2011; Gibelyou \& Huterer 2012; Rubart \& Schwarz 2013) and WENSS (Rubart \& Schwarz 2013) and was found within large error bars. While the direction of the observed radio dipole is consistent with the CMB dipole direction, its amplitude exceeds the theoretical expectations by a factor of a few. SKA will enable us to measure the radio dipole with high accuracy and to extract other low- $\ell$ multipole moments. Recently, the Planck mission reported a first detection of the effects of aberration and Doppler shift at high multipole moments (Planck collaboration 2014d). However, this observation is less precise than the reported measurements of the radio dipole and allows for a primordial contribution to the CMB dipole of comparable size.

The SKA will allow us to compare $\vec{d}_{\text {radio }}$ to $\vec{d}_{\mathrm{cmb}}$, since SKA will test a super-horizon sized volume. Any statistically significant deviation will be exciting, while finding a match would put the concordance model on firmer grounds.

SKA continuum surveys at low frequencies $(<1 \mathrm{GHz})$ should be ideal to probe the cosmic radio dipole already in the Early Science phase for two reasons. First, it is not necessary to cover the full area of the $3 \pi$ surveys, since a sparse sampling spread out over all of the accessible sky should be sufficient for a first estimate. And second, a focus on low frequencies and bright sources will pick primarily AGNs which have a much higher mean redshift than the SFG.

Figure 1 illustrates the accuracy that we can hope to achieve for a measurement of the radio dipole based on a linear estimator (Crawford 2009; Rubart \& Schwarz 2013). Our estimates are based on differential number counts from surveys in small and deep fields and simulations (Wilman et al. 2008). Our expectations for all-sky continuum surveys are summarized in Table 1. We find that the cosmic radio dipole can be measured at high statistical significance, even taking realistic data cuts into account (e.g. masking the galaxy and very bright extragalactic sources, or morphology, spectral index or flux cuts).

A major concern might be the effect of flux calibration errors on the dipole estimation. This has been studied by means of simulations. The results of this study are shown in figure 2 . We assume Gaussian flux density errors with variance $\sigma(\delta) S$, where $S$ denotes the expected flux density of a particular source and $\delta$ its declination. We consider the isotropic case in which $\sigma(\delta)=\sigma$ is isotropic and a declination dependent situation with $\sigma(\delta)=\sigma / \cos \left(\delta-\delta_{*}\right), \delta_{*}$ being fixed by 

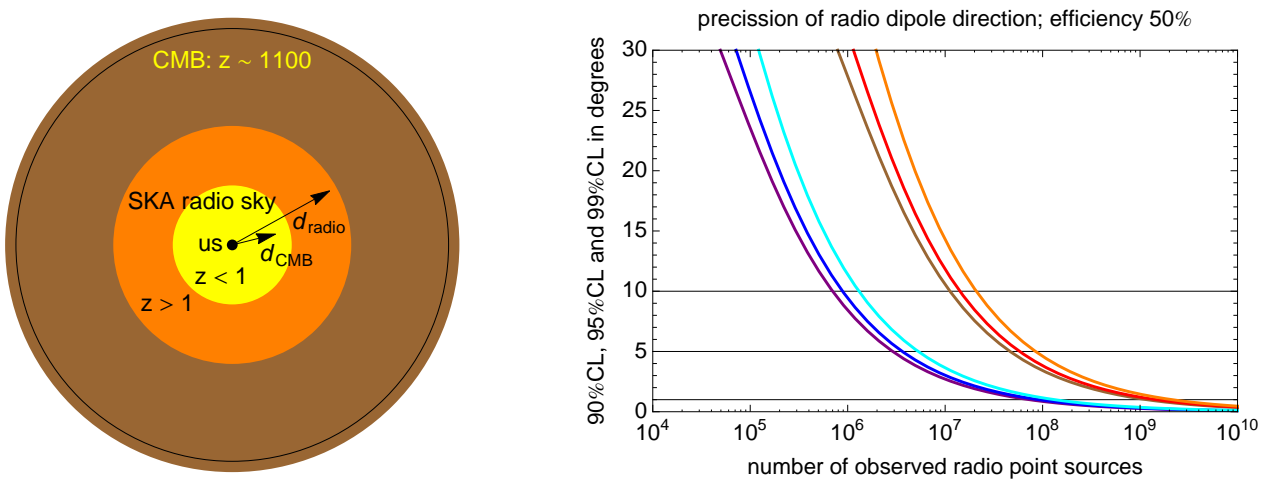

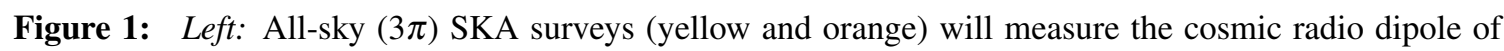
differential source counts. Selecting AGNs will result in a sample with median redshift $z>1$ (orange) and thus allow us to measure the peculiar velocity of the solar system with respect to the large scale structure on superhorizon scales. These measurements will be compared to the CMB dipole and thus test for the existence of a bulk flow of our Hubble volume compared to the CMB rest frame. Right: Angular accuracy at 90, 95 and $99 \%$ C.L. of the measurement of the cosmic radio dipole as a function of observed point sources. The blue set of curves assumes $d_{\text {radio }}=4 d_{\mathrm{cmb}}$, the red set assumes $d_{\text {radio }}=d_{\mathrm{cmb}}$. It is assumed that only $50 \%$ of all detected radio sources survive all quality cuts (e.g. masking fields that contain very bright sources). Combined with Table 1 we find that SKA Early Science allows detection of a possible deviation from the CMB expectation at high significance. SKA1 will constrain the cosmic radio dipole direction with an accuracy better than 5 degrees, SKA2 within a degree (at 99\% C.L.).
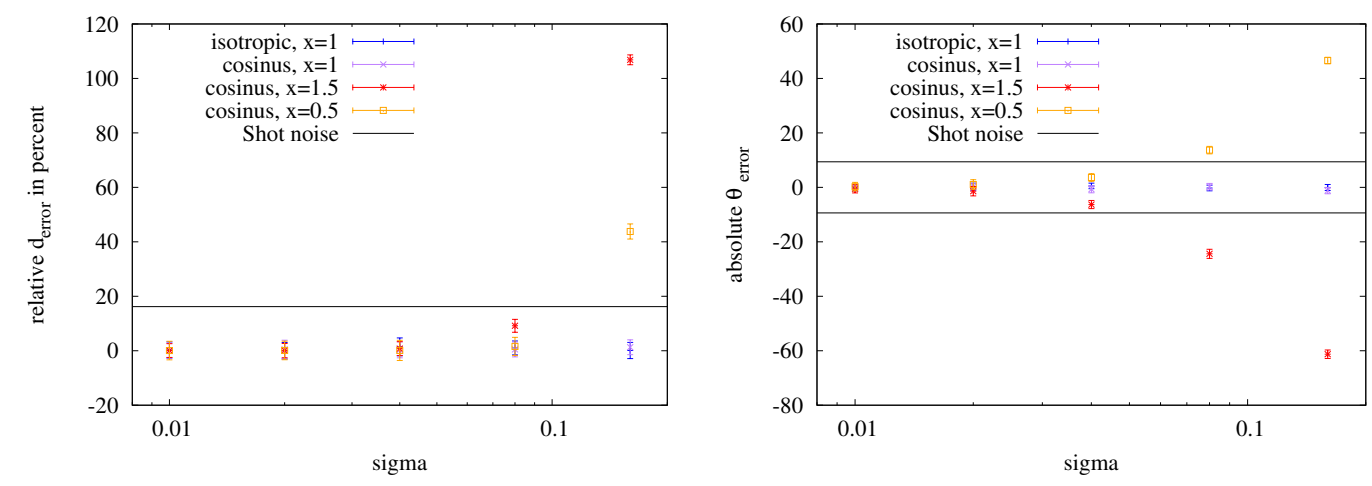

Figure 2: Left: Accuracy (in per cent) of the measurement of the dipole amplitude as function of fractional error on flux density calibration on individual point sources. All points are based on 100 simulations. Right: Accuracy (in degrees) of the measurement of the dipole direction. The horizontal lines denote the error due to shot noise for a dipole estimate based on $10^{7}$ sources (SKA Early Science).

the latitude of the SKA site and $\left|\delta-\delta_{*}\right|<70 \mathrm{deg}$. For two cases we find negligible influence of calibration errors: If the flux calibration error is completely isotropic or if the slope $x$ of the number counts $\left[N(>S) \propto S^{-x}\right]$ is equal to one. It turns out that $x=1$ is a special value, where calibration errors at the lower flux density limit have no influence on the dipole estimator. We conclude that direction dependent calibration effects must not exceed certain limits as shown figure 2.

Another significant contaminant of the kinetic radio dipole is the local structure dipole. We can turn a disadvantage of continuum surveys, namely that we observe several source populations, into an advantage as follows: The lower mean redshift of SFGs compared to AGNs allows us to change 


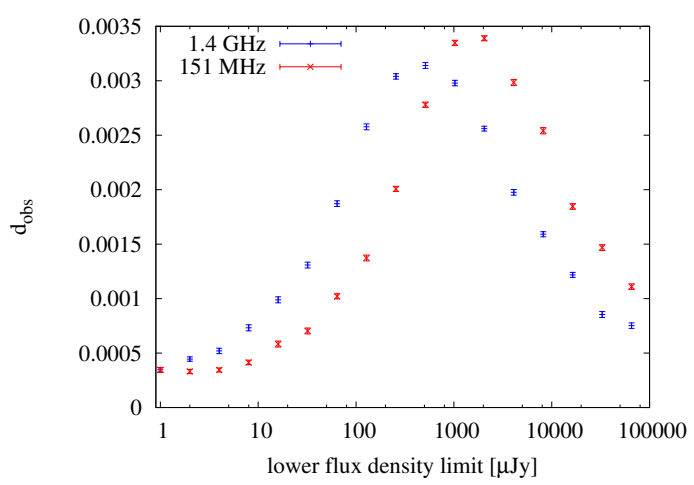

Figure 3: Amplitude of structure dipole due to a local void, affecting the measurements of the cosmic radio dipole as a function of lower flux density limit and for two wavebands centered on $151 \mathrm{MHz}$ and $1.4 \mathrm{GHz}$ (from Rubart et al. (2014)).

the mean depth of the survey by scanning different fluxes density limits and frequencies. This in fact allows for a tomographic survey of the radio dipole. For the example of a huge $(\sim 100 \mathrm{Mpc})$ local void this was studied recently (Rubart et al. 2014). Figure 3 illustrates this effect.

\section{Large angular scales (SKA1 \& SKA2)}

It is not obvious that the isotropic distribution of light implies also the isotropy of spacetime itself. The vanishing of the quadrupole and octopole moments of the CMB would imply the isotropy of space-time along the world line of the observer (Maartens 2011). While those low- $\ell$ multipoles are small compared to the monopole and dipole, they do not vanish exactly. We thus we can at best speak about an almost isotropic Universe. The radio sky offers another independent probe at $z>1$ and at the largest angular scales.

Recent work has revealed the existence of CMB "anomalies" [for a review, see Copi et al. (2010)]. In brief, the angular correlation function in the WMAP and Planck temperature maps vanishes on scales larger than 60 degrees, contrary to theoretical expectation; moreover, the CMB quadrupole and octopole anisotropy patterns are aligned both mutually and with respect to the Solar System geometry. These anomalies have been widely studied and discussed, but their origin remains unexplained.

SKA will provide a deep and wide large-scale structure dataset that will enable separating the effects of the early and late universe on the observed CMB anisotropy. For example, the SKA data could be used to reconstruct the late-time contribution to the CMB anisotropy via the integrated Sachs-Wolfe effect, and thus provide information about the temporal evolution of the CMB anomalies.

\subsection{Low- $\ell$ multipole moments}

The analysis of low- $\ell$ multipoles from SKA continuum surveys can benefit from the methods developed for the study of the CMB. Missing sky area is always a problem for low- $\ell$ mode measurements. For CMB studies, many methods were proposed to deal with a mask of missing data for both power spectrum estimation and phase recovery. For the power spectrum, one of the 

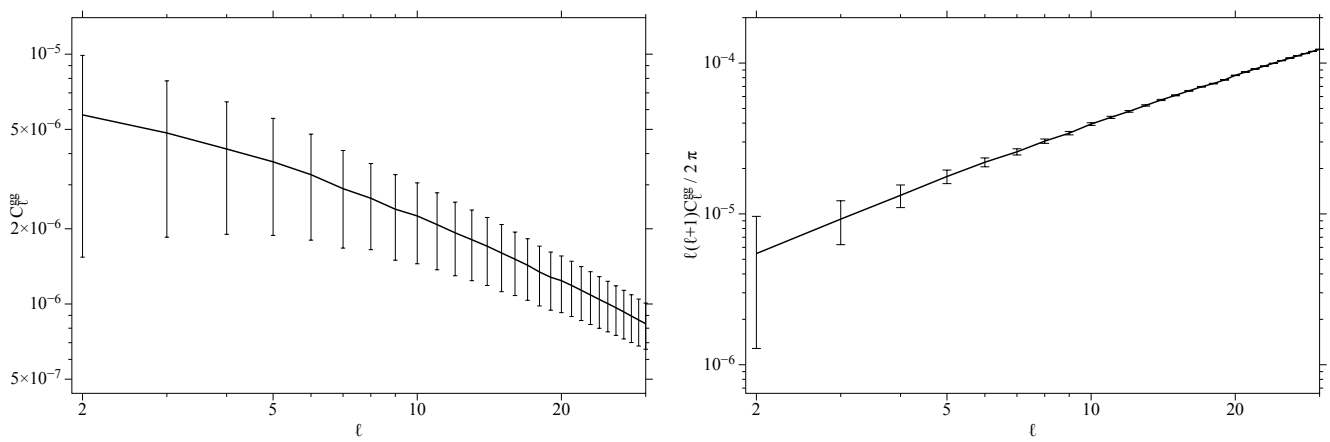

Figure 4: Left: Low- $\ell$ multipoles of the angular power spectrum as expected for a SKA1 continuum survey. Right: The corresponding band power. The errors contain shot noise and cosmic variance. The figures illustrate that statistically significant measurements of multipole moments can be expected.

most used methods is MASTER (Hivon et al. 2002). It consists in first building a matrix which captures the coupling between the modes induced by the mask, and then inverting this matrix. In Figure 4 we plot the angular power spectrum of SKA galaxies for low- $\ell$ mulitpoles with error bars corresponding to a SKA1 continuum survey.

For phase recovery, or more generally for large scale map reconstruction, many methods have been proposed based on Wiener filtering, $l_{2}$ or $l_{1}$ norm regularization, constraint realizations or diffusion [see Starck et al. (2013) and references therein]. Based on these new methodological idea, Planck data were analyzed with a mask removing $27 \%$ of the sky (Planck collaboration 2014c; Rassat et al. 2014). For a given observed sky area, the shape of the mask will also be important. The importance of random sampling is also described in Paykari et al. (2013), and many small missing parts, randomly distributed, will always be much better for large scale studies than a compact big missing part.

As for the dipole, SKA will tremendously improve the precision and quality of low- $\ell$ multipole moments and thus allow us to probe statistical isotropy, scale invariance and gaussianity.

\subsection{Angular 2-point correlation function}

The angular two point correlation is a powerful tool to measure the projected large-scale structure distribution of the Universe. It allows us to probe certain fundamental assumptions like scaleinvariance of the primordial perturbations, Gaussianity and the isotropy of the Universe (by comparing two-point correlations on sub-samples of the observed sky).

The two-point correlation at large angular scales contains many interesting aspects: Firstly, the matter fluctuations at large scales are in the linear regime. Secondly, general relativistic effects and cosmological evolution prefer large scales. AGNs are very good candidates to probe this, since they are isotropically distributed on the sky and most of them have a significant $(z>1)$ cosmological distance. In order to accurately investigate ultra-large scale correlations, the theoretical frame work of differential number counts based on general relativity will be needed (Maartens et al. 2013; Raccanelli et al. 2014, 2013; Chen \& Schwarz 2014).

In contrast to galaxy redshift surveys within the local Universe $(z \ll 1)$, all linear order relativistic corrections, which include the Doppler effect, lensing, and generalized Sachs-Wolfe contri- 
butions, are of relevance. The spread of luminosities of radio sources in continuum surveys washes out much of the clustering signal, and the general relativistic corrections are also suppressed. However, SKA HI surveys in which the source redshifts will be known will resolve these effects.

With the assistance of Lyman alpha data, one can model the luminosity function and evolution. With the SKA morphology data we expect to be able to identify different type of sources. This will allow us to study cross correlations between star forming galaxies and AGNs. We could also cross correlate with the CMB and different types of radio sources, which have different redshift distributions.

These aspects are treated in more detail in other contributions to this volume (Camera et al. 2015; Bull et al. 2015; Raccanelli et al. 2015). Let us just stress here the importance of reestablishing the almost scale-invariant power spectrum at superhorizon scales at $z \sim 1$, which will be possible by means of SKA all-sky surveys.

\section{Copernican Principle and homogeneity (SKA1 \& SKA2)}

The Copernican Principle is the assumption that we are not distinguished observers in the Universe. If we observe an isotropic cosmos, then distant observers should also see a similarly isotropic cosmos. This implies that the Universe satisfies the Cosmological Principle and is homogeneous on large scales. A violation of homogeneity in principle offers an alternative explanation to the acceleration of the Universe (Célérier 2000), but simple inhomogeneous models without dark energy are incompatible with current data (Bull et al. 2012). However, radial homogeneity is only weakly constrained in $\Lambda \mathrm{CDM}$ (Valkenburg et al. 2014). Any deviations would imply a radical change to the standard model and scale-invariant initial conditions, making it a vital constraint on the standard model.

SKA HI intensity mapping on super-Hubble scales offers powerful new ways to test homogeneity. By comparing the radial and transverse scale of baryon acoustic oscillations we can test isotropy of the expansion rate around distant observers (Maartens 2011; February et al. 2013; Clarkson 2012). This places direct constraints on radial inhomogeneity about us, when redshift-space distortions, lensing and other large-scale GR effects are accounted for. Anisotropic expansion rates act on the sound horizon at decoupling so that by redshift $z$ it has evolved into an ellipsoid with semi-axes

$$
L_{\|}(z)=\frac{\delta z(z)}{(1+z) H_{\|}(z)}, \quad L_{\perp}(z)=d_{A}(z) \delta \theta(z)
$$

given the observed radial and angular scales $\delta z(z), \delta \theta(z) . L_{\|}(z)=L_{\perp}(z)$ in a homogeneous universe. In an inhomogeneous universe $d_{A}(z)$ depends on the transverse Hubble rate along the line of sight, which will be different from the radial Hubble rate $H_{\|}(z)$, providing a test of homogeneity.

When combined with accurate distance data from SNIa, consistency relations can be used to check deviations from homogeneity in a completely model independent way (Clarkson et al. 2008). In a homogeneous universe, irrespective of dark energy or theory of gravity, the Hubble rate $h(z)=H(z) / H_{0}$ and dimensionless comoving distance $D(z)=(1+z) H_{0} d_{A}(z)$ satisfy $\left({ }^{\prime}=d / d z\right)$

$$
\mathscr{C}(z)=1+h^{2}\left(D D^{\prime \prime}-D^{\prime 2}\right)+h h^{\prime} D D^{\prime}=0
$$


so that $\mathscr{C}(z) \neq 0$ implies violation of the Copernican Principle. We expect that SKA1 will be able to constrain $\mathscr{C}(z)$ to $0 \pm 0.05$ for $z<1.5$, based on a naive error propagation from Bull et al. (2014). A more careful forecast has yet to be done. Direct constraints on radial inhomogeneity can be given combining with all available data sets which will significantly improve current constraints which are much weaker than those for isotropy (Valkenburg et al. 2014).

Finally, the Copernican Principle allows for the possibility of a fractal universe, but this is not predicted by the concordance model - which predicts a fractal dimension of 3 on large scales any deviations would imply new physics. It is therefore important to measure the fractal dimension of the distribution of radio sources at superhorizon scales. Such a test has been performed using the SDSS and the WiggleZ surveys, finding an approach to a three-dimensional distribution at $\sim 100$ Mpc scales (Hogg et al. 2005; Scrimgeour et al. 2012). A dramatic improvement will be possible based on SKA HI threshold surveys.

\section{Summary}

The Cosmological Principle provides the foundation for modern cosmology, and our understanding of the evolution of the Universe as well as all parameter constraints from the CMB, supernovae or large scale structure rely on this assumption. Testing the Cosmological Principle is thus of fundamental importance for cosmology generally as well as for the cosmological interpretation of the SKA data itself. As this chapter shows, SKA will be able to greatly increase our confidence that our cosmological framework makes sense (or lead to a scientific revolution if not).

We argue that SKA all-sky surveys will allow us to measure the cosmic radio dipole almost as precisely as the CMB dipole. SKA1 will constrain the cosmic radio dipole direction with an accuracy better than 5 degrees, SKA2 within a degree (at 99\% C.L.). This measurement could finally firmly establish or refute the commonly adopted assumption that the CMB and the overall LSS frames agree, and will have impact on a variety of cosmological observations, from the local measurement of $H_{0}$ to the calibration of CMB experiments. A tomography of the cosmic radio dipole might reveal a detailed understanding of local LSS.

In addition, studying the large-angular scales in SKA continuum and HI surveys might help resolve the puzzle of CMB anomalies and test the cosmological principle, including tests of statistical homogeneity. Further large-scale structure issues, especially non-Gaussianity and relativistic corrections, are discussed in Camera et al. (2015).

The ideas presented in this work only provide a flavor of SKA's potential to answer fundamental cosmological questions. Some of those ideas can already be tested by means of the SKA pathfinder experiments ASKAP, MeerKAT and LOFAR, but they cannot compete with SKA's survey speed and sensitivity. Thus SKA will be a unprecedented discovery and precision machine for modern cosmology.

\section{References}

Bennett, C. L., Larson, D., Weiland, J. L., et al. 2013, ApJS, 208, 20

Blake, C., \& Wall, J. 2002, Nature, 416, 150 
Braun, R. 2014, "SKA1 Imaging Science Performance", Document number SKA-TEL-SKO-DDXXX Revison A Draft 2

Bull, P., Camera, S., Raccanelli, A., et al. 2015, in "Advancing Astrophysics with the Square Kilometre Array", PoS(AASKA14)024

Bull, P., Clifton, T., \& Ferreira, P. G. 2012, Phys. Rev. D, 85, 024002

Bull, P., Ferreira, P. G., Patel, P., \& Santos, M. G. 2014, ArXiv e-prints, arXiv:1405.1452

Camera, S., Raccanelli, A., Bull, P., et al. 2015, in "Advancing Astrophysics with the Square Kilometre Array", PoS(AASKA14)025

Célérier, M.-N. 2000, A\&A, 353, 63

Chen, S., \& Schwarz, D. J. 2014, ArXiv e-prints, arXiv:1407.4682

Clarkson, C. 2012, Comptes Rendus Physique, 13, 682

Clarkson, C., Bassett, B., \& Lu, T. H.-C. 2008, Physical Review Letters, 101, 011301

Copi, C. J., Huterer, D., Schwarz, D. J., \& Starkman, G. D. 2010, Adv. Astron., 2010, 92

—. 2013a, ArXiv e-prints, arXiv:1310.3831

-. 2013b, ArXiv e-prints, arXiv: 1311.4562

Crawford, F. 2009, ApJ, 692, 887

de Zotti, G., Massardi, M., Negrello, M., \& Wall, J. 2010, A\&A Rev., 18, 1

Dewdney, P., Turner, W., Millenaar, R., et al. 2013, "SKA1 System Baseline Design”, Document number SKA-TEL-SKO-DD-001 Revision 1

Ellis, G. F. R., \& Baldwin, J. E. 1984, MNRAS, 206, 377

Erickcek, A. L., Carroll, S. M., \& Kamionkowski, M. 2008, Phys. Rev. D, 78, 083012

February, S., Clarkson, C., \& Maartens, R. 2013, JCAP, 3, 23

Gibelyou, C., \& Huterer, D. 2012, MNRAS, 427, 1994

Hinshaw, G., Weiland, J. L., Hill, R. S., et al. 2009, ApJS, 180, 225

Hivon, E., Górski, K. M., Netterfield, C. B., et al. 2002, ApJ, 567, 2

Hogg, D. W., Eisenstein, D. J., Blanton, M. R., et al. 2005, ApJ, 624, 54

Maartens, R. 2011, Roy. Soc. Lond. Phil. Trans. A, 369, 5115

Maartens, R., Zhao, G.-B., Bacon, D., Koyama, K., \& Raccanelli, A. 2013, JCAP, 2, 44

Paykari, P., Pires, S., Starck, J.-L., \& Jaffe, A. H. 2013, ArXiv e-prints, arXiv:1306.3035

Planck Collaboration, Ade, P. A. R., Aghanim, N., et al. 2014a, A\&A, 571, A16

-. 2014b, A\&A, 571, A22

-. 2014c, A\&A, 571, A23

Planck Collaboration, Aghanim, N., Armitage-Caplan, C., et al. 2014d, A\&A, 571, A27

Raccanelli, A., Bertacca, D., Doré, O., \& Maartens, R. 2014, JCAP, 8, 22

Raccanelli, A., Bertacca, D., Maartens, R., Clarkson, C., \& Doré, O. 2013, ArXiv e-prints, arXiv: 1311.6813

Raccanelli, A., Zhao, G.-B., Bacon, D. J., et al. 2012, MNRAS, 424, 801

Raccanelli, A., Bull, P., Camera, S., et al. 2015, in "Advancing Astrophysics with the Square Kilometre Array", PoS(AASKA14)031

Rassat, A., Starck, J.-L., Paykari, P., Sureau, F., \& Bobin, J. 2014, JCAP, 8, 6

Rubart, M., Bacon, D., \& Schwarz, D. J. 2014, A\&A, 565, A111

Rubart, M., \& Schwarz, D. J. 2013, A\&A, 555, A117

Ryle, M., \& Clarke, R. W. 1961, MNRAS, 122, 349 
Scrimgeour, M. I., Davis, T., Blake, C., et al. 2012, MNRAS, 425, 116 Singal, A. K. 2011, ApJL, 742, L23

Starck, J.-L., Fadili, M. J., \& Rassat, A. 2013, A\&A, 550, A15

Valkenburg, W., Marra, V., \& Clarkson, C. 2014, MNRAS, 438, L6

Wilman, R. J., Miller, L., Jarvis, M. J., et al. 2008, MNRAS, 388, 1335 